\title{
Proceso Enfermero aplicado a Paciente con Trastorno de Duelo
}

\section{Nursing Process applied to a Patient with Grief Disorder}

\author{
Emmanuel Arciniega-Rodríguez ${ }^{a}$, Norman A. Castillo-Hernández ${ }^{b}$, Daniel Juárez-Pérez ${ }^{c}$, \\ Paloma E. Meléndez-Orozco ${ }^{d}$, Luis G. Reyes-Pérez ${ }^{e}$, Cristian A. Revoreda-Montes ${ }^{f}$
}

\begin{abstract}
:
Grief is a common but complicated life experience, with a great risk of developing both physical and mental illnesses. In the literature it has been described after a debilitating clinical condition of a loss and recently it has been included in the Diagnostic and Statistical Manual of Mental Disorders (MMD-V) of the American Psychiatric Association. The work of the mental health nursing staff applied to grief is a complex process, which implies independent interventions and collaboration with other health professionals, such as psychiatry, psychology and thanatology, as it requires breaking the ties and ties contracted with the loved one, and face the pain of loss.
\end{abstract}

\section{Keywords:}

Grief, Mental Health, Nursing, Thanatology, Nursing Process

\section{Resumen:}

El duelo es una experiencia de vida común pero complicada, con un gran riesgo de desarrollar enfermedades tanto físicas como mentales. En la literatura se ha descrito una condición clínica debilitante después de una perdida y recientemente se ha incluido en el Manual Diagnóstico y Estadístico de los Trastornos Mentales (DSM-V) de la Asociación Americana de Psiquiatría. El trabajo del personal de enfermería en salud mental aplicado al duelo es un proceso complejo, que implica intervenciones independientes y de colaboración con otros profesionales de la salud, como psiquiatría, psicología y tanatología pues requiere deshacer los vínculos y lazos contraídos con el ser querido, y enfrentarse al dolor de la pérdida.

\section{Palabras Clave:}

Duelo, Salud mental, Enfermería, Tanatología, Proceso Enfermero

\section{Introducción}

El duelo, en sus raíces etimológicas proviene del latín dolus que quiere decir dolor, en este sentido la palabra lutos proveniente de la misma raíz tiene el significado de llorar. La palabra duelo y luto suelen ser palabras empleadas como sinónimos, aunque realmente el duelo hace referencia al proceso de cambios individuales significativos, en las emociones $y$ sentimientos, así como reacciones conductuales provocados por la pérdida o muerte de un ser querido, mientras que el luto hace referencia a la expresión social de las prácticas posteriores a la pérdida, estos son establecidos por el contexto social, es decir, los usos y costumbres, religión y espiritualidad.

El duelo es una experiencia de vida común pero

\footnotetext{
${ }^{a}$ Universidad Autónoma del Estado de Hidalgo, https://orcid.org/0000-0002-79469025, Email: ae315992@uaeh.edu.mx

b Universidad Autónoma del Estado de Hidalgo, https://orcid.org/0000-0001-8106-4126, Email: ca391317@uaeh.edu.mx

c Universidad Autónoma del Estado de Hidalgo, https://orcid.org/0000-0002-5121-0409, Email: daniel_juarez@uaeh.edu.mx

d Universidad Autónoma del Estado de Hidalgo, https://orcid.org/0000-0003-2687-6118, Email: me392144@uaeh.edu.mx

e Universidad Autónoma del Estado de Hidalgo, https://orcid.org/0000-0001-9067-0371, Email: re319435@uaeh.edu.mx

f Autor de Correspondencia, Universidad Autónoma del Estado de Hidalgo, https://orcid.org/0000-0001-5403-3527, Email: cristian_revoreda@uaeh.edu.mx
} 
complicada, con un gran riesgo de desarrollar enfermedades tanto físicas como mentales. En la literatura se ha descrito una condición clínica debilitante después de un duelo y recientemente se ha incluido en el Manual Diagnóstico y Estadístico de los Trastornos Mentales (DSM-V) de la Asociación Americana de Psiquiatría, un trastorno de duelo complejo persistente.

Esta condición ha sido previamente referida en la literatura como duelo complicado, que afecta aproximadamente al $7 \%-15 \%$ de las personas en duelo.

El trabajo del personal de enfermería en salud mental aplicado al duelo es un proceso complejo, que implica intervenciones independientes y de colaboración con otros profesionales de la salud, como psiquiatría, psicología y tanatología pues requiere deshacer los vínculos y lazos contraídos con el ser querido, y enfrentarse al dolor de la pérdida; es en esta actitud en la que la persona entra realmente en el proceso personal del duelo (1); estas intervenciones se plantean desde la perspectiva metodológica de la aplicación del Proceso Enfermero, lo que permite brindar cuidados de calidad, homogéneos, estandarizados y estructurados mediante la aplicación de las Taxonomías NANDA, NOC, y NIC; interrelacionando Guías de Práctica Clínica, Algoritmos de Atención Clínica, Enfermería Basada en Evidencias y la Normatividad aplicable en materia de Salud Mental. La enfermería es la disciplina que se encarga de atender las respuestas humanas que presenta el individuo a lo largo de su desarrollo, en este sentido el profesional de enfermería puede disminuir, y atender los efectos que el individuo puede manifestar a partir de una pérdida significativa.

\section{Presentación del caso}

Cristina, adulto medio, sexo femenino de 41 años, casada y tiene dos hijos ( $\mathrm{M}: 21$ y $\mathrm{H}: 13$ ), actualmente reside en Houston. Antes de la pandemia, era una mujer trabajadora, cuidaba niños (4-5 días a la semana), tenía una vida "normal", llevaba y recogía a su hija de la universidad, realizaba las tareas propias del hogar, le gustaba cocinar, llevaba a sus hijos a las actividades extraescolares (box). Ella practicaba Yoga. Con su esposo mantenía una buena relación, acompañándose y reforzándose en todo momento. Los lunes o viernes (días de descanso laboral) convivía con sus padres, aunque la madre solía visitarla esos días, el padre prefería no salir de casa. Durante la pandemia, ya no convivía tanto con sus padres, debido a las medidas sanitarias, solo los visitaba para llevarles víveres y cosas personales. El 13 de julio, se presenta la muerte de los padres de Cristina, ambos con diagnóstico de COVID-19 (fallecen el mismo día). El padre, adulto mayor de 77 años, aparentemente sano, es hospitalizado el día del padre (junio 2020), por presentar síntomas de COVID-19. Permaneció hospitalizado 20 días antes de su deceso. La madre, adulto mayor de 75 años, es hospitalizada una semana antes que el padre, además de presentar síntomas de Covid-19, era diabética e hipertensa. Durante la hospitalización presentó un paro cardiaco, motivo por el cual falleció. Cristina reside en Houston, ella es la hija número 4 de 5 hijos. Durante la hospitalización de los padres, la familia se mantuvo comunicada a través de llamadas, y videollamadas para tomar decisiones, ya que la comunicación con el personal médico fue limitada. Cristina y su familia, no pudieron despedirse de sus padres, por las restricciones sanitarias. Ella solo pudo realizar meditaciones y rituales para despedirse de ellos. No practica una religión en específico, pero suele visitar templos budistas, musulmanes, cabalistas y cristianas; cada hermano acompañó su proceso de luto de acuerdo con su religión. Como familia solo pudieron reunirse en la navidad pasada para honrar la memoria de sus padres. Tienen pensado hacer algo con las cenizas de sus padres para este año, ya que cumplirían 55 años de casados.

\section{Etapa I: Valoración por Patrones Funcionales.}

\section{Patrón I: Percepción-Mantenimiento de la salud:}

Manifestación de independencia: La paciente tiene conciencia de la muerte de sus padres (COVID) y de la pandemia en la que se encuentra, es capaz de pedir ayuda. Conoce acerca de la pandemia, y que es necesario tomar medidas específicas, como la distancia y no a la agrupación de personas.

No consume drogas ni abusa de fármacos. Suele realizar actividades con su marido como yoga.

Patrón II: Nutricional- metabólico:

En este patrón no refiere signos ni síntomas de alteración. Patrón III: Eliminación:

No refiere signos ni síntomas de alteración en este patrón funcional.

\section{Patrón IV: Actividad y ejercicio:}

Manifestación de independencia: Con su esposo mantiene una buena relación, practican yoga juntos y realiza actividades como cuidar niños (4-5 días a la semana) recogía a su hija de la universidad, cocinaba y llevaba a sus hijos a actividades extraescolares. No usa dispositivos de ayuda.

\section{Patrón V: cognitivo-perceptual:}

No refiere signos ni síntomas de alteración para este patrón funcional.

Patrón V: reposo-sueño:

Patrón sin alteraciones.

Patrón VII: autoimagen-autoconcepto:

Patrón sin alguna alteración

Patrón VIII: rol-relaciones: 
Refiere tener buena relación con su esposo en cuestión de realizar actividades recreativas y buena relación con sus hermanos para realizar la conmemoración por el aniversario de sus padres.

Patrón IX: Sexualidad/reproducción:

Existe buena relación con su marido.

Patrón X: Adaptación/Tolerancia al estrés:

Manifestación de independencia: Reconoce a su marido como un importante soporte familiar. No ha habido intentos de autolesión. El nivel de religiosidad es alto, ya que se apoya en la meditación y la religiosidad para encontrarse tranquila.

\section{Patrón XI: Valores/creencias:}

La religiosidad es la base fundamental para la tranquilidad personal.

No practica ninguna religión en específico, pero suele visitar templos budistas, musulmanes, cabalistas y cristianas, y realiza meditaciones y rituales.

\section{Etapa II: Diagnóstico.}

El diagnóstico enfermero es el juicio clínico sobre las respuestas humanas, individuales, grupales, familiares 0 de comunidad a problemas de salud reales o potenciales los cuales eventualmente requieren de intervenciones independientes o colaborativas con el propósito de resolverlas, disminuirlas o erradicarlas.

Los diagnósticos establecidos fueron:

1. Duelo.

El principal factor etológico la relación cercana con la persona fallecida y la dependencia.

Dentro de las características definitorias la paciente demostró mantener una conexión con la persona fallecida y la búsqueda constante de significado (de la muerte).

En este caso se determinó que la paciente se encuentra dentro de una población de riesgo pues ha pasado por la muerte de una persona significativa.

2. Disposición para mejorar los procesos familiares. El principal factor etológico la pérdida familiar.

Dentro de las características definitorias la paciente demostró deseos de mejorar la resiliencia familiar.

3. Disposición para mejorar el bienestar espiritual. El principal factor etológico la perdida familiar.

Dentro de las características definitorias la paciente demostró deseos de mejorar la práctica de la meditación, y mejorar la participación en actividades religiosas.

4. Disposición para mejorar el afrontamiento familiar. Dentro de las características definitorias la expresión de deseo de escoger experiencias que optimicen el bienestar.

5. Riesgo de deterioro de la resiliencia.

Dentro de las características definitorias la evidencia de procesos familiares disfuncionales y la ruptura de los rituales familiares.
En este caso de determino que la paciente se encuentra dentro de una población de riesgo ya que cuenta con datos demográficos que aumentan la probabilidad de desadaptación.

6. Riesgo de deterioro de la religiosidad.

Dentro de las características definitorias la evidencia de práctica religiosa interacción sociocultural insuficiente.

En este caso de determino que la paciente se encuentra dentro de una población de riesgo ya que esta en una crisis espiritual, personal y atraviesa por transiciones de vida.

A partir del diagnóstico enfermero se jerarquizaron los diagnósticos con el uso de la Red Lógica del Modelo de Análisis del Resultado del Estado Actual (Figura No.1). Con base a la utilización del modelo AREA, además de aplicar la premisa vida-función-estética dentro del proceso enfermero, los diagnósticos principales quedan de la siguiente manera:

1. Duelo relacionado con relación cercana con la persona fallecida 0 dependencia como lo demuestra crecimiento personal, mantener una conexión con el fallecido y búsqueda del significado.

2. Disposición para mejorar los procesos familiares relacionado con pérdida familiar como lo demuestra expresa deseo de mejorar la resiliencia familiar.

3. Disposición para mejorar el bienestar espiritual relacionado con pérdida familiar como lo demuestra deseo de mejorar la práctica de la meditación y mejorar la participación en actividades religiosas

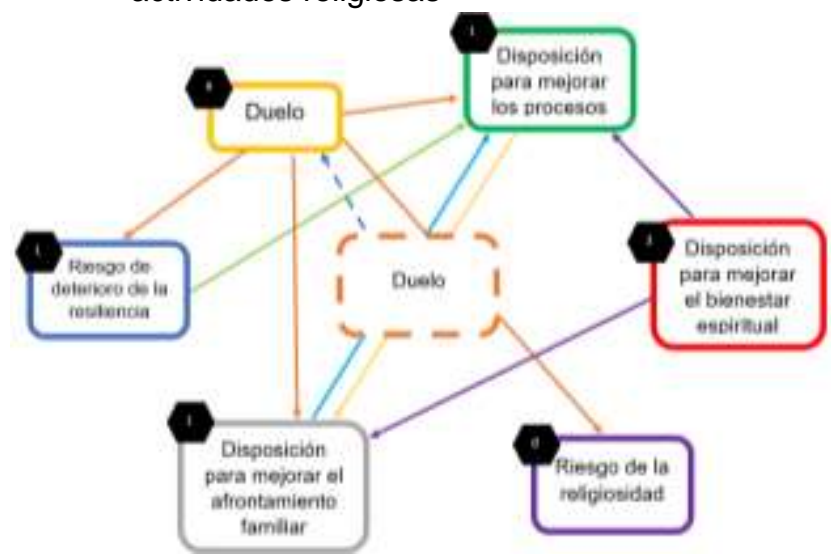

Figura No. 1 Jerarquización de los diagnósticos NANDA

\section{Etapa III y IV: Planeación y Ejecución}

En esta etapa se establecieron las estrategias necesarias para reforzar las respuestas humanas encontradas en la etapa de valoración, y estas determinaron cómo se brindarán los cuidados en el Proceso Enfermero. Para la implementación de estas intervenciones se requiere la 
colaboración de distintos enfoques multidisciplinarios (psicológicos y tanatológicos). A partir de la evaluación de las principales respuestas humanas, así como el establecimiento de las prioridades (diagnostico primario), basándose en la evidencia, los estándares relativos a la salud mental y la tanatología; se determinaron los problemas urgentes, y después los problemas generales. Para con base en estos datos, se estableciera el objetivo (NOC) a lograr en la paciente; mismo que dirigirá las intervenciones, así como los que son factores de protección para de la paciente.

Se estableció el resultado NOC, para medir la resolución del diagnóstico enfermero: Esperanza, perteneciente a al Dominio III: Salud psicosocial, y a la Clase M: Bienestar psicológico. Para ello se establecieron los siguientes indicadores:

1. Expresión de una orientación futura positiva

2. Expresión de optimismo

3. Establecimiento de objetivos

4. Expresión de confianza

5. Demostración de entusiasmo por la vida

A partir del objetivo NOC se plantearon las siguientes intervenciones NIC:

\section{Facilitar el duelo}

- Identificar la pérdida.

- Fomentar la expresión de sentimientos acerca de la pérdida (Escuchar las expresiones de duelo), de acuerdo con la Guía de Práctica Clínica de Cuidados Paliativos: Proporcionar información acerca del duelo y los recursos disponibles antes de la muerte y después de la muerte

- Realizar afirmaciones empáticas sobre el duelo, la identificación del riesgo de duelo complicado debería realizarse con base en:

- Factores de riesgo

- El juicio clínico

- La información acerca del entorno familiar

- Ayudar al paciente a identificar estrategias personales de afrontamiento.

- Fortalecer al paciente a implementar rituales de despedida, acompañados y asesorados de un tanatólogo (enfermera o psicólogo), apoyándose de las costumbres culturales, religiosas y sociales asociadas con la pérdida.

- Guiar para identificar las modificaciones necesarias del estilo de vida. $Y$ de ser necesario se recomienda derivar a psicología y a psiquiatría a las personas con duelo complicado según la GPC cuidados paliativos España, 2008.

\section{Dar esperanza}

- Informar al paciente acerca de si la situación actual constituye un estado temporal.

- Ayudar al paciente a expandir su yo espiritual. Estructurando un nuevo sentido de vida, con la aceptación de la ausencia.

- Fomentar las relaciones terapéuticas con los seres queridos, resignificación del ser querido.

- Considerar las intervenciones psicosociales, incluida la psicoterapia por el personal o equipo multidisciplinario, y si el caso lo requiere, por personal especializado según la GPC cuidados paliativos España, 2008

- Explicar a la familia los aspectos positivos de la esperanza (desarrollar temas de conversación que tengan sentido y que reflejan el amor y la necesidad del paciente) estableciendo redes de apoyo.

- En el duelo normal se recomienda proporcionar información acerca del duelo y sobre los recursos disponibles y apoyo emocional básico según la GPC cuidados paliativos, España 2008

- Crear un ambiente que facilite al paciente la práctica de su religión, cuando sea posible.

Una vez establecidas las intervenciones y validadas de acuerdo a los estándares de atención, se ejecutó el plan de cuidados, y la documentación de la evolución de la paciente, para esta etapa se puso a disposición de la paciente los recursos físicos, intelectuales, de referencia y contrareferencia, de tal forma que la paciente, familia, equipo de salud y redes de apoyo, con la dirección del personal de enfermería, se interviniera en las respuestas humanas identificadas en la etapa de valoración.

\section{Etapa V: Evaluación}

En esta etapa se realiza una valoración cuidadosa y detallada de los aspectos que ocurrieron durante todas las etapas de atención de la paciente, lo que permite continuar o concluir el proceso; para esto se realizó una evaluación preintervención mediante los indicadores NOC, encontrando una puntuación Diana de 3 , y se estableció la posibilidad de aumentar a 4, manteniendo en todo momento la puntación en un máximo de 3 puntos.

\begin{tabular}{|l|l|l|l|l|l|}
\hline \multicolumn{5}{|c|}{ Escalas } & $\mathbf{1}$ \\
\hline \multicolumn{1}{|c|}{ Indicadores } & $\mathbf{1}$ & $\mathbf{2}$ & $\mathbf{3}$ & $\mathbf{4}$ & $\mathbf{5}$ \\
\hline $\begin{array}{l}\text { Expresión de una } \\
\text { orientación futura positiva }\end{array}$ & & & $\mathbf{X}$ & & \\
\hline Expresión de optimismo & & & $\mathbf{X}$ & & \\
\hline $\begin{array}{l}\text { Establecimiento de } \\
\text { objetivos }\end{array}$ & & & $\mathbf{X}$ & & \\
\hline Expresión de confianza & & $\mathbf{X}$ & & & \\
\hline $\begin{array}{l}\text { Demostración de } \\
\text { entusiasmo por la vida }\end{array}$ & & $\mathbf{X}$ & & & \\
\hline
\end{tabular}


Una vez ejecutadas las intervenciones se realizó una evaluación posintervención encontrando una puntuación Diana de 4 , lo que sugiere continuar con el proceso terapéutico y eventualmente aumentar a 5 puntos.

\begin{tabular}{|l|l|l|l|l|l|}
\hline \multicolumn{4}{|c|}{ Escalas } & & \\
\hline \multicolumn{1}{|c|}{ Indicadores } & $\mathbf{1}$ & $\mathbf{2}$ & $\mathbf{3}$ & $\mathbf{4}$ & $\mathbf{5}$ \\
\hline $\begin{array}{l}\text { Expresión de una } \\
\text { orientación futura positiva }\end{array}$ & & & & $\mathbf{X}$ & \\
\hline Expresión de optimismo & & & $\mathbf{X}$ & & \\
\hline $\begin{array}{l}\text { Establecimiento de } \\
\text { objetivos }\end{array}$ & & & & $\mathbf{X}$ & \\
\hline Expresión de confianza & & & $\mathbf{X}$ & & \\
\hline $\begin{array}{l}\text { Demostración de } \\
\text { entusiasmo por la vida }\end{array}$ & & & & $\mathbf{X}$ & \\
\hline
\end{tabular}

\section{Conclusiones}

En muchas ocasiones resulta complicado recolectar datos, aún más cuando los sistemas sanitarios están saturados y las condiciones epidemiológicas precisan distanciamiento social, si bien el duelo, como proceso humano, no se trata de una enfermedad, se ha tornado uno de los desafíos más imponentes para el profesional de enfermería quien debe establecer una relación terapéutica en el que la empatía y confianza sean el principal eslabón que una a la persona con el profesional. Esto pone de manifiesto que el Proceso Enfermero con el personal de enfermería como director puede acoger al paciente como un ser biopsicosocial y espiritual, que permite individualizar los cuidados poniendo en práctica el juicio reflexivo, que ayude a alcanzar el máximo nivel de independencia de los pacientes.

\section{Recomendaciones}

- Asegurar que la familia pueda tener acceso a grupos de apoyo o especialistas en salud mental.

- Motivar a la paciente a conversar a través de la meditación u oraciones, con sus seres fallecidos.

- Sugerir uso de técnicas de distracción o alto al pensamiento para combatir pensamientos intrusivos sobre la muerte o la persona fallecida

- Implementar técnicas de relajación para situaciones de ansiedad o insomnio.

- Llevar a cabo un ritual de despedida que permita el cierre de círculos, y la conclusión de las celebraciones religiosas a distancia.

- Reincorporar a la paciente a sus actividades ordinarias de forma eventual, ordenada y de acuerdo a sus posibilidades (distanciamiento social propio de la pandemia) además de motivarla al encuentro familiar-social a través de dispositivos móviles.
NOTA: En duelos complicado: se realizarán intervenciones psicoterapéuticas específicas y se establecerá un diagnostico diferencial entre duelo y depresión mayor y/o el uso apropiado de psicofármacos si se requiere.

\section{Referencias}

1. Cuidados Paliativos GUÍA DE PRÁCTICA CLÍNICA gpc CUIDADOS PALIATIVOS Evidencias y recomendaciones Catálogo maestro de guías de práctica clínica: IMSS-440-11 [Internet]. ; $\quad$ Available from:http://www.cenetec.salud.gob.mx/descargas/gpc/Catalogo Maestro/445_GPC_Cuidados_paliativos/GER_Cuidados_Paliat ivosx 1x.pd $\mathrm{f}$

2. DUELO Manual de Capacitación para Acompañamiento y Abordaje de Duelo. Duelo Manual de Capacitación para Acompañamiento y Abordaje de Duelo [Internet]. ; Available from:

https://www.unicef.org/elsalvador/media/3191/file/Manual\%20 so bre\%20Duelo.pd

3. Mexicana De Tanatología A. El duelo y su proceso para superarlo. Tesina que para obtener el diplomado en tanatología presenta: esperanza díaz maldonado [Internet]. ; Available from: http://www.tanatologia

amtac.com/descargas/tesinas/64\%20El\%20duelo $\% 20 \mathrm{y} \% 20 \mathrm{su} \%$ 20 proceso.pdf

4. Fernández Liria, A, Rodríguez Vega, B. Intervenciones sobre problemas relacionados con el duelo para profesionales de Atención Primaria (I): el proceso del duelo. Medifam [Internet]. 2021 [cited 2021 May 7];12(3):100-7. Available from: https://scielo.isciii.es/scielo.php?script=sci_arttext\&pid=S1131 $-57682002000300008$

5. Mesquita AC, Costa Valcanti Avelino C, Neves Barreto M, Alves Nogueira D, Souza Terra F de, Cássia Lopes Chaves é de. El bienestar espiritual y la prestación del cuidado espiritual en un equipo de enfermería. Index de Enfermería [Internet]. 2014 Dec [cited 2021 May 7];23(4):219-23. Available from: https://scielo.isciii.es/scielo.php?script=sci_arttext\&pid=S1132 - 12962014000300006

6. Julián C, Vargas P. Spirituality as a Means for Human Development A espiritualidade como meio de desenvolvimento humano [Internet]. ; 2012. Available from: http://www.scielo.org.co/pdf/cteo/v42n98/v42n98a09.pdf

7. Herdman TH, Kamitsuru S. NANDA International Nursing Diagnoses: Definitions \& Classification, 2018-2020. 11a ed. Herdman TH, editor. New York, NY: Thieme Medical; 2017.

8. Butcher HK, Bulechek GM, Dochterman JM, Wagner C, editores. Clasificación de Intervenciones de Enfermería (NIC). 7a ed. Elsevier; 2018.

9. Moorhead S, Johnson M, Maas ML, editores. Clasificación de Resultados de Enfermería (NOC). 6a ed. Elsevier Health Sciences; 2019.

10. Roch JLG. Enfermería psiquiátrica. Elsevier; 2016.

11. Rodríguez S. BA. PROCESO DE ENFERMERÍA APLICACIÓN ACTUAL ED.3. CUELLAR AYALA; 2015 
Publicación semestral, Educación y Salud Boletín Científico Instituto de Ciencias de la Salud Universidad Autónoma del Estado de Hidalgo, Vol. 9, No. 18 (2021) 136-141

12. Iglesias, JA. “¿Hacia dónde va el proceso enfermero de la tercera generación?" RevistaEnfermeríaCyL 9.2 (2017): 1-2.

13. Sevilla, Juan Carlos R. "Papel de enfermería en el juicio clínico: la valoración y el diagnóstico." Enfermería en cardiología: revista científica e informativa de la Asociación Española de Enfermería en Cardiología 62 (2014): 25-31

Haga clic o pulse aquí para escribir texto. 